\title{
Author Correction: A high-energy and long-cycling lithium-sulfur pouch cell via a macroporous catalytic cathode with double-end binding sites
}

Chen Zhao, Gui-Liang Xu(D), Zhou Yu, Leicheng Zhang, Inhui Hwang, Yu-Xue Mo, Yuxun Ren, Lei Cheng (D),

Cheng-Jun Sun, Yang Ren (D), Xiaobing Zuo (D), Jun-Tao Li, Shi-Gang Sun, Khalil Amine (D) and Tianshou Zhao (D)

Correction to: Nature Nanotechnology https://doi.org/10.1038/s41565-020-00797-w, published online 23 November 2020.

In the version of this Article originally published online, in affiliation 8, 'IRMC' should have been 'Institute for Research \& Medical Consultations'; this has now been corrected in all versions.

Published online: 3 December 2020

https://doi.org/10.1038/s41565-020-00829-5

(c) UChicago Argonne, LLC, Operator of Argonne National Laboratory under exclusive licence to Springer Nature Limited 2020 